\title{
An Electron-Transfer Path through an Extended Disulfide Relay System: The Case of the Redox Protein ALR
}

\author{
Lucia Banci, ${ }^{*},+\ddagger$ Ivano Bertini, ${ }^{*}, \dagger$ Vito Calderone, ${ }^{\dagger, \dagger}$ Chiara Cefaro, ${ }^{\dagger, \dagger}$ Simone Ciofi-Baffoni, ${ }^{\dagger, \dagger}$ \\ Angelo Gallo, ${ }^{\dagger, \ddagger}$ and Kostas Tokatlidis ${ }^{\perp, \|}$ \\ ${ }^{\dagger}$ Magnetic Resonance Center, University of Florence, via L. Sacconi 6, Sesto Fiorentino, Italy \\ ${ }^{\ddagger}$ Department of Chemistry, University of Florence, via della Lastruccia 3, Sesto Fiorentino, Italy \\ ${ }^{\perp}$ Institute of Molecular Biology and Biotechnology, Foundation for Research and Technology Hellas, Heraklion 70013, Crete, Greece \\ "Department of Materials Science and Technology, University of Crete, Heraklion 71003, Crete, Greece
}

Supporting Information

ABSTRACT: The oxidative folding mechanism in the intermembrane space of human mitochondria underpins a disulfide relay system consisting of the import receptor Mia40 and the homodimeric FAD-dependent thiol oxidase ALR. The flavoprotein ALR receives two electrons per subunit from Mia40, which are then donated through oneelectron reactions to two cytochrome $c$ molecules, thus mediating a switch from two-electron to one-electron transfer. We dissect here the mechanism of the electron flux within ALR, characterizing at the atomic level the ALR intermediates that allow electrons to rapidly flow to cytochrome $c$. The intermediate critical for the electrontransfer process implies the formation of a specific intersubunit disulfide which exclusively allows electron flow from Mia40 to FAD. This finding allows us to present a complete model for the electron-transfer pathway in ALR.

$\mathrm{M}$ ia40 traps proteins in the intermembrane space (IMS) of mitochondria via an oxidative folding mechanism. ${ }^{1-3} \mathrm{~A}$ large share of the IMS proteins, produced by the ribosome in the cytosol, reach the IMS in a largely unfolded state and are folded through the Mia40-catalyzed formation of disulfide bonds, ${ }^{4-7}$ thereby becoming trapped in the IMS. Mia40 has been therefore defined as a hub for protein oxidation. ${ }^{8}$ After interaction with its substrates, the active site of Mia40 protein, a CPC motif, becomes reduced. ${ }^{9,10}$ Its oxidized, functional state is then restored by a flavin adenine dinucleotide (FAD)dependent sulfhydryl oxidase, named augmenter of liver regeneration (ALR) in humans. ${ }^{11}$ ALR takes electrons from Mia40, which is thus reoxidized, and delivers them to cytochrome $c$ (cyt $c)^{12,13}$ (Figure 1 ).

ALR forms head-to-tail homodimers. Each subunit consists of a core domain of 100 amino acid residues organized as a four-helix bundle that binds a FAD molecule, and a flexible segment of 80 amino acid residues at the $\mathrm{N}$-terminus. ${ }^{13-16} \mathrm{~A}$ conserved redox-active $\mathrm{CX}_{2} \mathrm{C}$ motif forms an active-site disulfide located proximal to the isoalloxazine ring of the $\mathrm{FAD}$ cofactor (called proximal disulfide). The flexible $\mathrm{N}$ terminal tail segment contains another conserved $\mathrm{CX}_{2} \mathrm{C}$ motif, called distal disulfide. ${ }^{17}$ It has been shown previously that the core domain of ALR containing only the proximal disulfide is

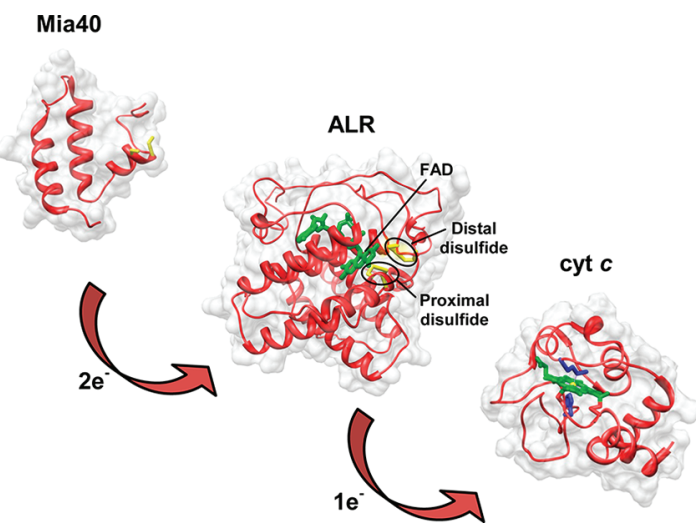

Figure 1. Redox centers of Mia40, ALR, and cyt $c$, shown within the frame of their structures in the electron flow cascade responsible for the oxidative folding mechanism in the IMS. The flavoprotein ALR mediates the switch from two-electron to one-electron transfer, receiving two electrons per subunit from Mia40 and donating them one at a time to two cyt $c$ molecules. The cysteines of the reduced CPC active site of Mia40 as well as the distal and proximal disulfides of ALR are shown in yellow; FAD and heme cofactors in ALR and cyt $c$, respectively, are in green; and the His and Met axial ligands of the iron ion (shown as a yellow sphere) of cyt $c$ are in blue.

sufficient to oxidize small substrates like dithiothreitol. ${ }^{12}$ The proximal disulfide receives electrons directly from the substrate, transferring them to the nearby $\mathrm{FAD}$ moiety. From $\mathrm{FAD}$, the electrons are passed on to downstream electron acceptors, i.e., cyt $c$ or oxygen. ${ }^{12}$ For larger molecules, like Mia40, both the distal and proximal disulfides are required for their oxidation, and the two subunits of the homodimer cooperate in an intersubunit electron transfer. ${ }^{11}$

As part of our efforts to characterize ALR in humans, we have dissected here the route of electron flux from Mia40 to cyt $c$ through ALR at the atomic level. We have specifically characterized the ALR intermediates that allow the electrons to efficiently flow from the active CPC site of Mia40 to the FAD moiety of ALR, passing through the distal and proximal disulfides. To this end we have used either an ALR construct

Received: October 20, 2011

Published: January 6, 2012 
(ALR 65-205) containing the distal disulfide but devoid of the largely unfolded 1-64 N-terminal segment, ${ }^{13}$ or a shorter one containing only the proximal disulfide (ALR 81-205).

The electron-transfer cascade from reduced Mia40 to oxidized cyt $c$ in a reconstituted system with purified components is relatively fast, as cyt $c$ is stoichiometrically reduced in less than $1 \mathrm{~min}$ (Supporting Information (SI), Figure S1). When the Cys residues of the distal and proximal disulfides in each ALR subunit (Figure 1) are absent or mutated to Ser (see methods in the SI), the electron transfer from Mia40 to cyt $c$ is kinetically impaired and occurs at the same low level as when ALR itself is completely absent from the reaction (Figure S1). We had already shown that the Cys71Cys74 disulfide bond (distal disulfide) of ALR is specifically recognized by Mia40, ${ }^{13}$ and therefore it constitutes the entry point for the two electrons flowing from the reduced CPC motif of Mia40 to ALR. The electron-transfer mechanism within ALR was then investigated by characterizing selected Cys $\rightarrow$ Ser mutants of the distal and proximal disulfides. By mutating Cys145 together with either Cys71 or Cys74, a disulfide bridge between the other two remaining Cys, i.e., one from each disulfide pair, is formed (Figure S2). If, however, Cys142 is mutated together with either one of the cysteines of the distal pair, no disulfide bond is formed (Figure S2). These data indicate that the electron flow from the distal to the proximal disulfide depends crucially on Cys142 and must involve a transient disulfide pair between Cys142 and either Cys 71 or Cys74, forming two possible intermediates indicated as $\mathbf{b}$ and $\mathbf{b}^{\prime}$ in Figure 2.

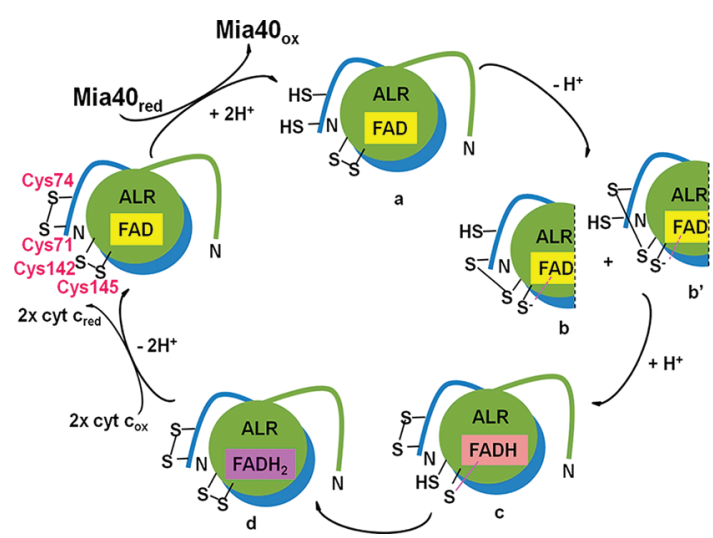

Figure 2. Electron-transfer mechanism in ALR, shown for only one subunit for clarity. The substrate Mia40 reduces the distal disulfide of ALR (the two ALR subunits are shown in green and blue), generating the $\mathbf{a}$ intermediate, which evolves in the $\mathbf{b}$ and $\mathbf{b}^{\prime}$ intermediates, having a disulfide bond between Cys142 and either one of the cysteines of the distal pair, and Cys145 in a thiolate state. Nucleophilic attack of Cys 145 thiolate on the C4a of FAD generates the flavin C4a-Cys145 thiol intermediate $\mathbf{c}$, which finally evolves into the intermediate $\mathbf{d}$, having FADH2. From FADH2, two electrons are passed, one at a time, to two cyt $c$ molecules.

Both of these intermediates contain oxidized FAD, as shown from their corresponding electronic absorption spectra (Figure $3 A)$. Furthermore, ALR with the distal disulfide in the reduced state is the only intermediate ( $\mathrm{a}$ in Figure 2) able to allow the electrons to flow from Mia40 to FAD. In fact, no interaction is observed between Mia40 and the two ALR mutants having a disulfide bond between the distal and proximal motifs (Figure S3). This also indicates that Mia40 recognizes the ALR N-
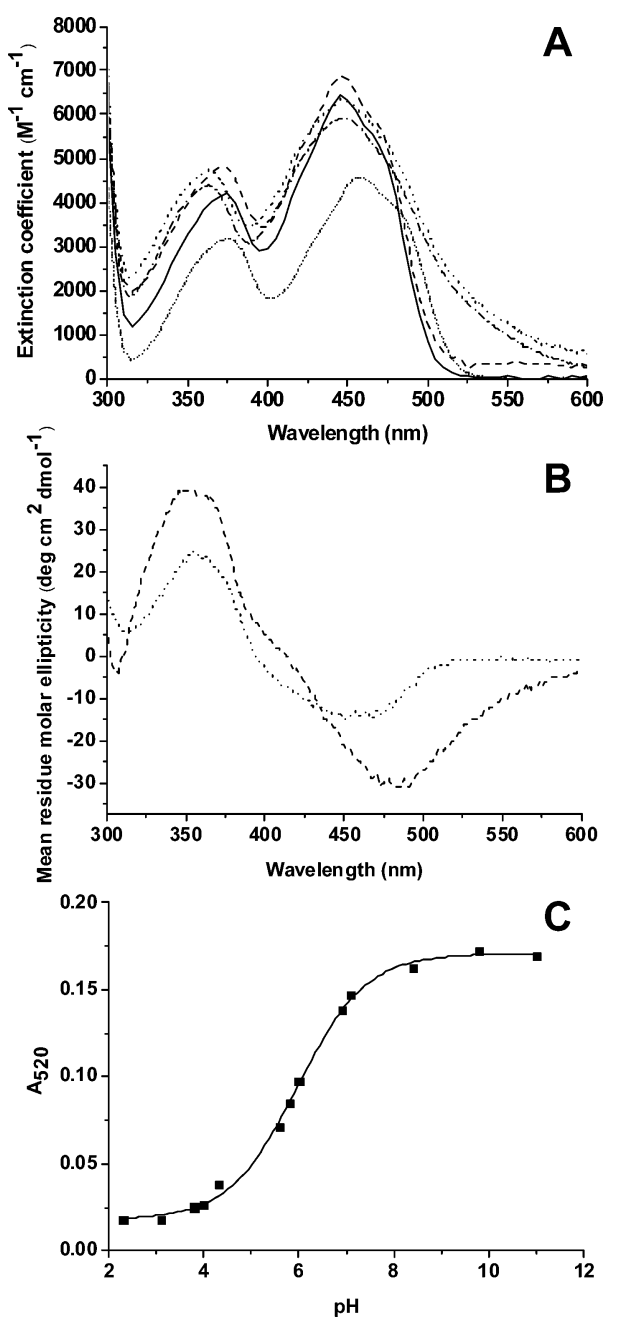

Figure 3. UV-visible (A) and circular dichroism (B) absorption spectra of ALR 65-205 mutants (in panel A, - Cys71/145Ser, --Cys74/145Ser, ... Cys71/142Ser, -.- Cys74/142Ser, ... wild-type; in panel B, ... Cys145Ser, --- Cys142Ser). (C) pH titration of Cys142Ser ALR $81-205$. The absorbance at $520 \mathrm{~nm}$ is plotted as a function of $\mathrm{pH}$. The solid line shows the fit profile according to a single $\mathrm{pK}_{\mathrm{a}}$ equation.

terminal segment containing the distal disulfide only when the latter can freely move in solution without any restriction imposed by any intersubunit disulfide bond, which also agrees with the previous report that the N-terminal segment of Erv1, the yeast homologue of ALR, can interact with Mia40, even when added as an isolated peptide in trans. ${ }^{18}$

In order to describe the interaction between FAD and Cys145 in the $\mathbf{b}$ and $\mathbf{b}^{\prime}$ intermediates, the crystal structure at $\mathrm{pH} 7.2$ of a construct containing only Cys145 (see methods in the SI) was solved at $1.9 \AA$ resolution. The structure is totally superimposable with that of the wild-type protein (PDB ID 3O55), except for the mutated cysteine. This mutant shows the absence of covalent bonds between Cys 145 and the FAD ring. However, the distance between $S \gamma$ of Cys145 and C4a of the $\mathrm{FAD}$ ring (the reactive carbon of flavins ${ }^{19,20}$ ) is $3.0 \AA$, i.e., compatible with a charge-transfer interaction (Figure 4). Indeed, electronic and circular dichroism spectra show a charge-transfer band extending from $470 \mathrm{~nm}$ to beyond 550 $\mathrm{nm}$, centered at about $490 \mathrm{~nm}$, which accounts for the orange color of this mutant and the mutants missing Cys142 (Figure 


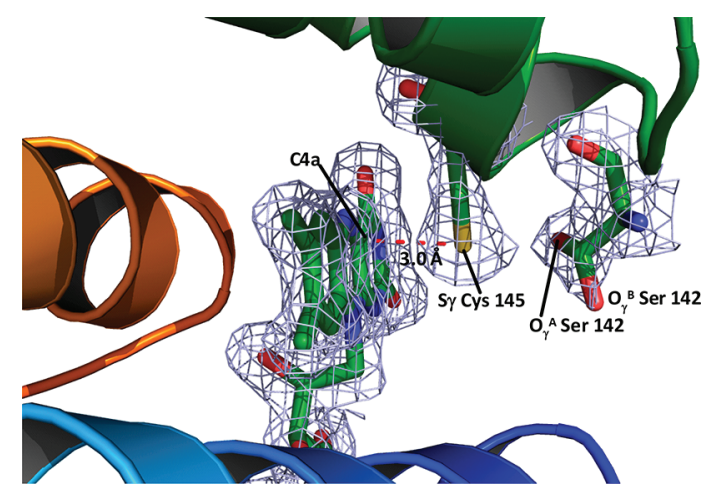

Figure 4. Detailed view of the Cys142Ser ALR 81-205 mutant showing the interaction between $\mathrm{C} 4 \mathrm{a}$ of $\mathrm{FAD}$ and $\mathrm{S} \gamma$ of Cys 145 and the double conformation (A and B) of the side chain of Ser142. The electron density of the above-mentioned residues is also shown. The distance between the $\mathrm{C} 4 \mathrm{a}$ position of the isoalloxazine ring and the sulfur atom of Cys145 is indicated.

3A,B). This charge-transfer band is absent in the yellow-colored wild-type protein (Figure 3A), in which the $S \gamma$ of Cys145 is at a distance of $3.3 \AA$ from the $\mathrm{C} 4 \mathrm{a}$ of FAD. Both crystal and absorption spectral data therefore suggest the presence of a thiolate state for Cys 145 which interacts with the oxidized FAD (b and $\mathbf{b}^{\prime}$ intermediates of Figure 2). As reported in the literature, ${ }^{21-26}$ it appears indeed that only the thiolate state of a cysteine can be a charge-transfer donor to oxidized FAD. According to that, a $\mathrm{p} K_{\mathrm{a}}$ value of $5.95 \pm 0.07$ was calculated for the $S \gamma$ of Cys 145 (see SI and Figure 3C), in agreement with the unusually low $\mathrm{pK}_{\mathrm{a}}$ values of a Cys thiolate in redox communication to $\mathrm{FAD}$ in a number of flavoenzymes. ${ }^{27,28}$ The crystal structure of the mutant without both Cys142 and Cys145 was also solved, showing that the distance between the $\mathrm{O} \gamma$ of Ser145 and C4a of FAD goes back to the wild-type-like value $(3.2 \AA)$. As a result of the sulfur-to-oxygen substitution, this mutant is yellow-colored, with no observable chargetransfer absorption band. In both crystal structures, the oxygen atom of Ser142 shows a very mobile side chain with at least two conformers (Figure 4) and a poor electron density, while it also becomes more solvent exposed due to the loss of the disulfide bond with Cys145. This accounts for our finding that Cys142 is the only cysteine of the proximal disulfide that is capable of forming the disulfide bond with the distal pair ( $\mathbf{b}$ and $\mathbf{b}^{\prime}$ intermediates of Figure 2).

In conclusion, we have shown that the electron flow from Mia40 to the FAD moiety of ALR takes place through the formation of a first ALR intermediate (a in Figure 2) released from Mia40 with the distal cysteines in a fully reduced state. This then evolves into two possible intermediates ( $\mathbf{b}$ and $\mathbf{b}^{\prime}$ in Figure 2) having a disulfide bond between Cys142 and any of the distal pair, and with Cys 145 in a thiolate state stabilized by a charge transfer from $\mathrm{S} \gamma$ of Cys 145 to $\mathrm{C} 4 \mathrm{a}$ of FAD. According to all reported catalytic mechanisms of $\mathrm{FAD}$-dependent reduc$\operatorname{tases}^{21,27}$ and dehydrogenase, ${ }^{29}$ the nucleophilic attack of Cys 145 thiolate to the $\mathrm{C} 4 \mathrm{a}$ of $\mathrm{FAD}$ can generate the flavin C4aCys 140 thiol intermediate (c in Figure 2), which accounts for the transfer of two electrons from the proximal cysteines to oxidized FAD. FADH2 (d in Figure 2) then transfers one electron at a time to two molecules of cyt $c$.

\section{ASSOCIATED CONTENT}

\section{Supporting Information}

Supporting table and figures; details about protein production and characterization, crystallization, and structure determination. This material is available free of charge via the Internet at http://pubs.acs.org.

\section{AUTHOR INFORMATION}

\section{Corresponding Author}

banci@cerm.unifi.it; ivanobertini@cerm.unifi.it

\section{ACKNOWLEDGMENTS}

This work was supported by the Access to Research Infrastructures activity in the 7th Framework Programme of the EC (Bio-NMR Contract 261863), and by the Italian MIUR-FIRB PROTEOMICA RBRN07BMCT (to I.B. and L.B.) and funds from IMBB-FORTH, the University of Crete, and the European Social Fund and national resources (to K.T.).

\section{REFERENCES}

(1) Chacinska, A.; Pfannschmidt, S.; Wiedemann, N.; Kozjak, V.; Sanjuan Szklarz, L. K.; Schulze-Specking, A.; Truscott, K. M.; Guiard, B.; Meisinger, C.; Pfanner, N. EMBO J. 2004, 23, 3735.

(2) Mesecke, N.; Terziyska, N.; Kozany, C.; Baumann, F.; Neupert, W.; Hell, K.; Herrmann, J. M. Cell 2005, 121, 1059.

(3) Naoe, M.; Ohwa, Y.; Ishikawa, D.; Ohshima, C.; Nishikawa, S. I.; Yamamoto, H.; Endo, T. J. Biol. Chem. 2004, 279, 47815.

(4) Sideris, D. P.; Tokatlidis, K. Antioxid. Redox Signal. 2010, 13, 1189.

(5) Deponte, M.; Hell, K. J. Biochem. 2009, 146, 599.

(6) Gross, D. P.; Burgard, C. A.; Reddehase, S.; Leitch, J. M.; Culotta, V. C.; Hell, K. Mol. Biol. Cell 2011, 22, 3758.

(7) Riemer, J.; Fischer, M.; Herrmann, J. M. Biochim. Biophys. Acta 2011, 1808, 981.

(8) Banci, L.; Bertini, I.; Cefaro, C.; Cenacchi, L.; Ciofi-Baffoni, S.; Felli, I. C.; Gallo, A.; Gonnelli, L.; Luchinat, E.; Sideris, D. P.; Tokatlidis, K. Proc. Natl. Acad. Sci. U.S.A. 2010, 107, 20190.

(9) Banci, L.; Bertini, I.; Cefaro, C.; Ciofi-Baffoni, S.; Gallo, A.; Martinelli, M.; Sideris, D. P.; Katrakili, N.; Tokatlidis, K. Nat. Struct. Mol. Biol. 2009, 16, 198.

(10) Terziyska, N.; Grumbt, B.; Kozany, C.; Hell, K. J. Biol. Chem. 2009, 284, 1353.

(11) Daithankar, V. N.; Farrell, S. R.; Thorpe, C. Biochemistry 2009, 48, 4828.

(12) Farrell, S. R.; Thorpe, C. Biochemistry 2005, 44, 1532.

(13) Banci, L.; Bertini, I.; Calderone, V.; Cefaro, C.; Ciofi-Baffoni, S.; Gallo, A.; Kallergi, E.; Lionaki, E.; Pozidis, C.; Tokatlidis, K. Proc. Natl. Acad. Sci. U.S.A. 2011, 108, 4811.

(14) Daithankar, V. N.; Schaefer, S. A.; Dong, M.; Bahnson, B. J.; Thorpe, C. Biochemistry 2010, 49, 6737.

(15) Wu, C. K.; Dailey, T. A.; Dailey, H. A.; Wang, B. C.; Rose, J. P. Protein Sci. 2003, 12, 1109.

(16) Vitu, E.; Bentzur, M.; Lisowsky, T.; Kaiser, C. A.; Fass, D. J. Mol. Biol. 2006, 362, 89.

(17) Fass, D. Biochim. Biophys. Acta 2008, 1783, 557.

(18) Lionaki, E.; Aivaliotis, M.; Pozidis, C.; Tokatlidis, K. Antioxid. Redox Signal. 2010, 13, 1327.

(19) Neims, A. H.; Hellerman, L. Annu. Rev. Biochem. 1970, 39, 867.

(20) Miura, R. Chem. Rec. 2001, 1, 183.

(21) Miller, S. M.; Massey, V.; Ballou, D.; Williams, C. H. Jr.; Distefano, M. D.; Moore, M. J.; Walsh, C. T. Biochemistry 1990, 29, 2831.

(22) Hoober, K. L.; Thorpe, C. Methods Enzymol. 2002, 348, 30.

(23) Hoober, K. L.; Thorpe, C. Biochemistry 1999, 38, 3211.

(24) Coppock, D. L.; Thorpe, C. Antioxid. Redox Signal. 2006, 8, 300.

(25) Dmitrenko, O.; Thorpe, C. J. Sulfur Chem. 2008, 29, 415. 
(26) Hoober, K. L.; Joneja, B.; White, H. B. III; Thorpe, C. J. Biol. Chem. 1996, 271, 30510.

(27) Williams, C. H. J. Lipoamide dehydrogenase, glutathione reductase, thioredoxin reductase, and mercuric ion reductase-A family of flavoenzyme transhydrogenases. In Chemistry and Biochemistry of Flavoenzymes; Muller, F., Ed.; CRC Press: Boca Raton, FL, 1992; pp $121-211$.

(28) Thorpe, C.; Hoober, K. L.; Raje, S.; Glynn, N. M.; Burnside, J.; Turi, G. K.; Coppock, D. L. Arch. Biochem. Biophys. 2002, 405, 1.

(29) Thorpe, C.; Williams, C. H. Jr. J. Biol. Chem. 1976, 251, 7726. 